\title{
Energization of particles in Saturn's inner magnetosphere: Monte Carlo simulation of stochastic electric field effects
}

\author{
E. Martínez-Gómez, H. Durand-Manterola, and H. Pérez
}

\begin{abstract}
Institute of Geophysics, Department of Space Physics, National University of Mexico, Ciudad Universitaria 04510, Mexico e-mail: affabeca@gmail.com; hdurand_manterola@yahoo.com;perezdet@geofisica.unam.mx
\end{abstract}

Received 10 October 2006 / Accepted 23 April 2007

\begin{abstract}
Context. Our knowledge of energetic particles in Saturn's inner magnetosphere is based on observations made during the flybys of Pioneer 11, Voyager 1, Voyager 2, and recently by Cassini. The most important features of the energetic particle population in the inner Saturnian magnetosphere are: 1) the rings and the many large and small satellites inside this region reduce the population of particles whose energies are higher than $0.5 \mathrm{MeV}$ to values of the order of $10^{3}$ times less than would otherwise be present; 2) the sputtering and outgassing of the surfaces of the satellites injects particles into the system and by some physical process, particles of the resultant plasma are accelerated to energies of the order of tens of $\mathrm{keV} ; 3$ ) the radial distribution of very energetic protons $E_{\mathrm{p}}>$ tens of $\mathrm{MeV}$ exhibits three major peaks associated with rings and satellites; 4) a proton population $E_{\mathrm{p}} \sim 1 \mathrm{MeV}$ lies outside the orbit of Enceladus; 5) a proton population $E_{\mathrm{p}}<0.25 \mathrm{MeV}$ has an apparent origin associated with Dione, Tethys, Enceladus, E-ring, Mimas, and G-ring; 6) a population of low-energy electrons is associated with the satellites.

Aims. We propose a mechanism to explain the energetic particle population observed in Saturn's inner magnetosphere based on the stochastic behavior of the electric field.

Methods. To simulate the stochastic electric field we employ a Monte Carlo Method taking into account the magnetic field fluctuations obtained from the observations made by Voyager 1 spacecraft.

Results. Assuming different initial conditions, like the source of charged particles and the distribution function of their velocities, we find that particles injected with very low energies ranging from $0.104 \mathrm{eV}$ to $0.526 \mathrm{keV}$ can be accelerated to reach much higher energies ranging from $0.944 \mathrm{eV}$ to $0.547 \mathrm{keV}$ after a few seconds.
\end{abstract}

Key words. acceleration of particles - magnetic fields - plasmas - methods: statistical - planets and satellites: general

\section{Introduction}

The available observations in situ of Saturn's magnetosphere were made by the Pioneer 11 (September 1979), Voyager 1 (November 1980), and Voyager 2 (August 1981) spacecraft. In July 2004, Cassini arrived at Saturn and is now providing information on the structure and dynamics of this magnetosphere.

Pioneer 11 provided information of energetic ions $(100 \mathrm{eV}-8 \mathrm{keV})$ at distances $4-16 R_{\mathrm{S}}\left(1 R_{\mathrm{S}}=60268 \mathrm{~km}\right)$ from Saturn and within $1 R_{\mathrm{S}}$ of the equatorial plane (Frank et al. 1980). These data pointed to a dense $\left(>1 \mathrm{~cm}^{-3}\right)$ and rigidly co-rotating magnetosphere out to a distance of $10 R_{\mathrm{S}}$, but slightly less beyond this distance. The maximum ion number densities $\left(\sim 50 \mathrm{~cm}^{-3}\right)$ were measured near the orbits of Tethys and Dione, indicating that they were surrounded by plasma torii around Saturn.

Later, during the Voyager encounters the state of the magnetosphere was more quiescent but also with significant temporal variations between and during the encounters (Krimigis et al. 1983). The Voyager 1 and 2 trajectories were steeper in latitude past Saturn, but the Plasma Spectrometer (PLS) onboard both Voyager (able to detect energetic particles from $10 \mathrm{eV}$ to $6 \mathrm{keV}$ ) nevertheless confirmed the largely co-rotating behavior of the magnetosphere beyond $4 R_{\mathrm{S}}$ (see for example, Bridge et al. 1982; Sittler et al. 1983; Richardson 1986). Additionally, the magnetosphere was found to be populated by low-energy (soft) electrons in the outer region and more energetic electrons close in. Substantially higher counting rates of protons $\left(E_{\mathrm{p}}>80 \mathrm{MeV}\right)$ were observed inside the orbits of Enceladus and Mimas, indicative of very energetic particles in the radiation belts of Saturn. The energy spectra of ions change inside the magnetosphere as a function of radial distance as well as a function of local time. The shape of these ion energy spectra can be described by a Maxwellian distribution function for lower energies and by a power-law distribution function for energies above a few hundred $\mathrm{keV}$. Krimigis et al. (1982), found a characteristic temperature based on these spectra as $6 \times 10^{8} \mathrm{~K}$ (or about $55 \mathrm{keV}$ ). Two-component proton spectra were found in Saturn's inner magnetosphere (Krimigis \& Armstrong 1982), a low-energy population $(<500 \mathrm{keV})$ described by a power-law with index 2.5 , and a high-energy part $(>16 \mathrm{MeV})$ with a spectral form similar to that expected from cosmic ray neutron albedo decay (CRAND) (Fillius \& Mcllwain 1980; Krimigis et al. 1981). This is indicative of different sources of particles present in the Saturnian system: the solar wind, the planetary ionosphere, Titan, the icy satellites surfaces and their tiny atmospheres, and the rings (Richardson et al. 1986; Jurac et al. 2002; Krupp 2005; Krimigis et al. 2005; Sittler et al. 2006). The rings and the many large and small satellites in the inner magnetosphere reduce the population of particles whose energies are higher than $0.5 \mathrm{MeV}$ to values of the order of $10^{3}$ times less than would otherwise be present, in other words, the rings and satellites can absorb 
energetic particles. Some of these absorption microsignatures were reported by Simpson et al. (1980), Roussos et al. (2005), and Paranicas et al. (2005).

The Cassini spacecraft reached on its orbit injection (SOI) trajectory much deeper into the magnetosphere of Saturn and crossed the equatorial plane twice just outside the F-ring at $2.5 R_{\mathrm{S}}$. The closest approach was only $1.3 R_{\mathrm{S}}$ from Saturn's center above the visible rings. Wahlund et al. (2005) found a dense $\left(<150 \mathrm{~cm}^{-3}\right)$ and cold $(<7 \mathrm{eV})$ plasma torus just outside the visible F-ring. This torus of partly dusty plasma does not co-rotate perfectly with Saturn, which suggests the cold plasma is electrodynamically coupled to the charged ring-dust particles. The cold ion characteristics changed near the magnetically conjugate position of Dione, indicating the release of volatile material from this icy moon.

Basically, Saturn's magnetosphere can be divided into three regions of plasma characterized by different physical and chemical properties. They are: a) the outer magnetosphere containing hot plasma dominated by protons and its velocity varies between 30 and $80 \%$ of the rigid corotation speed, b) a region that consists of partially corotating plasma and made up of a mixture of protons, $\mathrm{O}^{+}, \mathrm{OH}^{+}, \mathrm{H}_{2} \mathrm{O}^{+}$and $\mathrm{H}_{3} \mathrm{O}^{+}$and, c) the inner magnetosphere that consists of plasma closer to rigid corotation and contains protons, $\mathrm{O}^{+}, \mathrm{OH}^{+}, \mathrm{H}_{2} \mathrm{O}^{+}$and $\mathrm{N}^{+}$. Young et al. (2005) has proposed a new region, the thin layer of plasma located directly over the $\mathrm{A}$ and $\mathrm{B}$ rings and composed of $\mathrm{O}^{+}$ and $\mathrm{O}_{2}^{+}$.

Because Saturn is much like Earth and Jupiter in its dynamical properties, similar solar wind-driven and/or rotational responses are expected. It is not clear yet that dayside reconnection will lead to substorms and then to the acceleration of particles, but there are some recent reports of pulsating dayside auroral features on Jupiter (Gladstone et al. 2002), of small scale transient Earth-like reconnection signatures at Jupiter (Grodent et al. 2004), of extreme auroral variability at Saturn (Grodent 2004), of bursts of ion activity in the tail (Mitchell et al. 2005), and of energetic particle injections in Saturn's magnetosphere (Mauk et al. 2005) which could indicate this type of activity in Saturn's magnetosphere. Besides the reconnection mechanism to accelerate particles, other mechanisms have been proposed to explain the energization of particles in planetary magnetospheres. For example, charged particles spiraling along magnetic field lines are accelerated through resonant interactions with plasma waves (Abe \& Nishida 1986; Barbosa 1986). A variety of mechanisms are explained in the works of Blandford (1994), Treumann \& Pottelette (2002) and Kivelson (2005).

To explain the observations of energetic particles in Saturn's magnetosphere, Schardt et al. (1985) proposed that the release of magnetic energy heats the ion component of the plasma and then accelerates electrons to energies of about $2 \mathrm{MeV}$. Krimigis et al. $(1981,1982)$ supposed that changes in the tail configuration induced by interplanetary disturbances may lead to the acceleration of both ions and electrons to several hundred $\mathrm{keV}$. In this work we propose a model in which the equation of motion includes the force due to the stochastic electric field derived from the magnetic field fluctuations measured by Voyager 1 spacecraft within Saturn's inner magnetosphere, the Lorentz force, the corotational electric field force and the gravitational force. We developed a numerical algorithm to solve this equation under several initial conditions like the source of ions (Saturn's ionosphere, the C-ring, the south pole of Enceladus, Dione's icy surface, the E-ring and Rhea) and their velocity distributions. We find that the high-energy part $(>1 \mathrm{keV})$ of the final spectrum for ions follows a power-law distribution, and the low-energy part
$(<1 \mathrm{keV})$ can follow an asymmetric sigmoidal, an exponential decay or a logistic distribution. As a consequence the plasma is heated to reach higher temperatures and can be transported to the outer regions of the magnetosphere.

\section{Description of the energization model for Saturn's inner magnetosphere}

When a stochastic force acts on the particles, there is a preference to increase the particle's velocity (instead of slowing them down). This fact has been demonstrated by Durand-Manterola (2003). Hence, the observed population of energetic particles in a planetary magnetosphere can be explained in terms of the action of this stochastic field. In this work we consider that a stochastic electric field acts on the population of charged particles in Saturn's inner magnetosphere.

To quantify the gain of energy per particle we developed a numerical algorithm which solves the equation of motion and basically works as follows: from a sample of charged particles that initially follow a velocity distribution and an energy distribution we randomly choose one of them and make it interact with the stochastic electric field (simulated by a Monte Carlo method). After one interaction the final velocity, the final position, and the final energy of such particle are calculated (those values become the initial parameters for the next interaction). When the particle has undergone the chosen number of interactions, another particle is randomly selected and then injected into the simulated magnetosphere. This new particle undergoes the same process described before. The algorithm finishes when all the particles have undergone the same number of interactions.

\subsection{Equation of motion for a single charged particle}

A single charged particle with mass $m$ and charge $q$ which is injected throughout the magnetosphere is governed by the following equation of motion:

$$
\frac{\mathrm{d}^{2} \boldsymbol{r}}{\mathrm{d} t^{2}}=\frac{q}{m} \boldsymbol{E}_{\mathrm{STOC}}+\frac{q}{m}\left(\boldsymbol{E}_{\mathrm{C}}+\frac{\mathrm{d} \boldsymbol{r}}{\mathrm{d} t} \times \boldsymbol{B}\right)-G \frac{M_{\mathrm{S}}}{\left|\boldsymbol{r}^{3}\right|} \boldsymbol{r} .
$$

Equation (1) is written in MKS units and we are assuming an inertial coordinate system fixed to Saturn's center of mass. The particle's position vector is denoted by $\boldsymbol{r}$. The first term on the right side in Eq. (1) represents the stochastic acceleration, the second term on the right is the Lorentz acceleration, and the last term is the gravitational acceleration. For this work we have taken the usual meanings and values for all the fundamental physical constants.

\subsubsection{The stochastic acceleration}

The magnetic field within a planetary magnetosphere has two components: $\boldsymbol{B}_{\mathrm{f}}$ is the magnetic field generated by the planetary dynamo (it is constant and very intense) and $\boldsymbol{B}_{\mathrm{e}}$ are the temporal magnetic field fluctuations generated by the changes in the interaction between the solar wind and the magnetosphere (they are stochastic and less intense). A stochastic electric field, $\boldsymbol{E}_{\text {STOC }}$, based on those fluctuations is obtained through Faraday's law.

In this work we use the magnetic field data averaged every $1.92 \mathrm{~s}$ by the triaxial fluxgate magnetometer carried by the Voyager 1 spacecraft and the trajectory position data with a resolution of $3 \times 10^{4} \mathrm{~m}$. The measurements are given in 


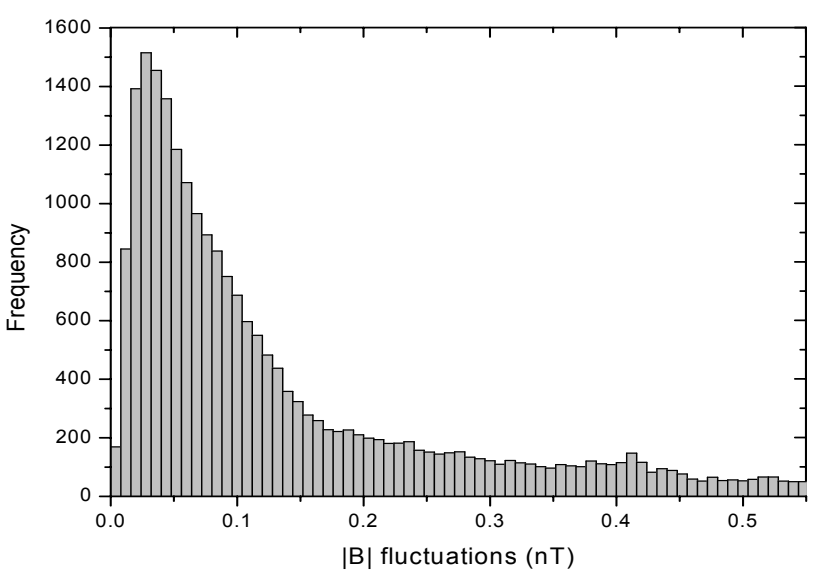

Fig. 1. Histogram of frequencies for the magnetic field fluctuations, $\Delta \boldsymbol{B}$, obtained in Saturn's inner magnetosphere from the first inbound to the fifth outbound crossings through the magnetopause. Voyager 1 spacecraft crossed the magnetosphere from November 12, 1980 at 01:54 UT to November 14, 1980 at 21:40 UT.

the Saturn Solar Orbital Coordinate System (SSO). Voyager 1 moved through the magnetosphere from November 12, 1980 at 01:54 UT to November 14, 1980 at 21:40 UT. We will consider the first inbound magnetopause crossing and the fifth outbound magnetopause crossing as boundaries of the magnetosphere (Bridge et al. 1981; Ness et al. 1982).

The magnetic field observed by Voyager 1 has two components: spatial and temporal. If we assume that the spatial magnetic field of Saturn is well represented by a dipole of moment $0.21 \pm 0.005$ Gauss- $R_{\mathrm{S}}^{3}$ (Ness et al. 1981), we might calculate the residual magnetic field, $\boldsymbol{B}_{\mathrm{R}}$, at a particular point of the particles trajectory:

$\boldsymbol{B}_{\mathrm{R}}=\boldsymbol{B}_{\mathrm{O}}-\boldsymbol{B}_{\mathrm{D}}$

where $\boldsymbol{B}_{\mathrm{O}}$ is the observed magnetic field and $\boldsymbol{B}_{\mathrm{D}}$ is the dipolar magnetic field. In our study the residual magnetic field only has temporal variations. Then the magnetic field fluctuations, $\Delta \boldsymbol{B}$, are calculated by taking into account the differences of adjacent data points of the residuals.

To obtain the dipolar planetary magnetic field we take the expressions derived by Kivelson \& Russell (1997) and for Saturn we estimate the value of the dipolar moment as $3.96 \mathrm{~T} \mathrm{~m}^{3}$. In Fig. 1 we show the histogram for the temporal magnetic field fluctuations in Saturn's inner magnetosphere based on Voyager 1 magnetic field observations.

From the Maxwell equations we can obtain the associated electric field in each coordinate $(x, y, z)$. Assuming charge quasineutrality, i.e. $\rho=0$, and that the changes of the magnetic field throughout the spacecraft trajectory are small enough $(\Delta \approx \partial)$, we can solve them and get:

$$
\begin{aligned}
& \Delta E_{x}=\frac{\frac{\Delta B_{z}}{\Delta t}\left(\frac{\Delta x_{\mathrm{T}}}{\Delta y_{\mathrm{T}}}+\frac{\Delta x_{\mathrm{T}} \Delta y_{\mathrm{T}}}{\left(\Delta z_{\mathrm{T}}\right)^{2}}\right)+\frac{\Delta B_{x}}{\Delta t} \frac{\Delta y_{\mathrm{T}}}{\Delta z_{\mathrm{T}}}}{\frac{1}{\Delta x_{\mathrm{T}}}+\Delta x_{\mathrm{T}}\left(\frac{1}{\left(\Delta y_{\mathrm{T}}\right)^{2}}+\frac{1}{\left(\Delta z_{\mathrm{T}}\right)^{2}}\right)} \\
& \Delta E_{y}=\Delta x_{\mathrm{T}}\left(\frac{\Delta E_{x}}{\Delta y_{\mathrm{T}}}-\frac{\Delta B_{z}}{\Delta t}\right) \\
& \Delta E_{z}=\Delta x_{\mathrm{T}}\left(\frac{\Delta E_{x}}{\Delta z_{\mathrm{T}}}+\frac{\Delta B_{y}}{\Delta t}\right) .
\end{aligned}
$$

In Eqs. (3)-(5), the difference between adjacent points on the spacecraft trajectory is given by $\Delta x_{\mathrm{T}}, \Delta y_{\mathrm{T}}$, and $\Delta z_{\mathrm{T}}$. The cases
Table 1. Values of the parameters $\alpha$ and $\beta$ for the Voyager 1 spacecraft during its encounter with Saturn's inner magnetosphere occurred from November 12, 1980 at 15:31 UT to November 13, 1980 at 07:59 UT.

\begin{tabular}{lcc}
\hline \hline$\Delta E$ component & & \\
$\left(\mathrm{V} \mathrm{m}^{-1}\right)$ & $\alpha$ & $\beta$ \\
\hline$\Delta E_{x}$ & $6.35 \times 10^{-7}$ & $6.05 \times 10^{-4}$ \\
$\Delta E_{y}$ & $2.31 \times 10^{-6}$ & $4.22 \times 10^{-4}$ \\
$\Delta E_{z}$ & $-6.84 \times 10^{-5}$ & $3.81 \times 10^{-3}$ \\
\hline
\end{tabular}

when $\Delta x_{\mathrm{T}}=\Delta y_{\mathrm{T}}=\Delta z_{\mathrm{T}}=0$ do not have any mathematical sense, thus the value of the electric field is not considered. This fact does not affect the distribution of the data because we apply the same time sampling, $\Delta t(1.92 \mathrm{~s})$.

To simulate the stochastic behavior of the electric field obtained through Eqs. (3)-(5) we use a Monte Carlo Method. Basically, for this method we need the probability distribution functions (or pdf's) that describe the physical system under study and then it proceeds by random sampling of such pdf's.

In order to find that density function, we do a goodness-of-fit hypothesis test to the data obtained through Eqs. (3)-(5) using the value of the Anderson-Darling (AD) statistical criterion at the 95\% significance level (Stephens 1974) obtained for the normal, exponential, logistic, lognormal, Weibull, and the extreme value probability distributions. The decision rule for this hypothesis test establishes that a smaller value of the AD statistic indicates that the distribution fits the data better. In our case the pdf is given by the Logistic distribution function.

Once we get the adequate pdf we choose the sampling rule from the following: random number generator, sampling from analytic pdf's and sampling from tabulated pdf's. Because of the mathematical nature of the Logistic distribution function it is possible to obtain the cumulative distribution function and then proceed by the direct inversion of such function. In this way we obtain the stochastic electric field for each component from:

$\triangle E(\xi)=\alpha+\beta \log _{\mathrm{e}}\left(\frac{\xi}{1-\xi}\right)$

where $\alpha$ is the expected value of this distribution function whereas $\beta$ is given in terms of the variance as: $\sigma^{2}=3.29 \beta^{2}$ and $\xi$ is a random number uniformly distributed on the interval $[0,1]$. The values of $\alpha$ and $\beta$ calculated for the Voyager 1 data are shown in Table 1.

When we apply Eq. (6) we are considering that the stochastic electric field spectrum (Fig. 2) is the same for all latitudes and local time within Saturn's inner magnetosphere because it is derived from the induced fluctuations of the solar wind on the magnetosphere through the magnetic field fluctuations shown in Fig. 1.

\subsubsection{The Lorentz acceleration}

In Eq. (1) the second term on the right corresponds to the Lorentz force. The rotation of Saturn's magnetic field induces an electric field in the radial direction: the corotational electric field, $\boldsymbol{E}_{\mathrm{C}}$. For a neutral particle or an observer in the magnetosphere, the magnetic field moves with a corotation velocity, $\boldsymbol{v}_{\mathrm{C}}$, $\boldsymbol{v}_{\mathrm{C}}=\omega_{\mathrm{ROT}} \times \boldsymbol{r}$ with $\omega_{\mathrm{ROT}}$ the spin angular velocity of Saturn with a value of $1.638 \times 10^{-4} \mathrm{rad} \mathrm{s}^{-1}$ and $\boldsymbol{r}$ the distance. This induces an electric field given by:

$\boldsymbol{E}_{\mathrm{C}}=-\left(\boldsymbol{v}_{\mathrm{C}} \times \boldsymbol{B}_{\mathrm{D}}\right)=\left(\boldsymbol{r} \times \omega_{\mathrm{ROT}}\right) \times \boldsymbol{B}_{\mathrm{D}}$

with $\boldsymbol{B}_{\mathrm{D}}$ the dipolar magnetic field. 


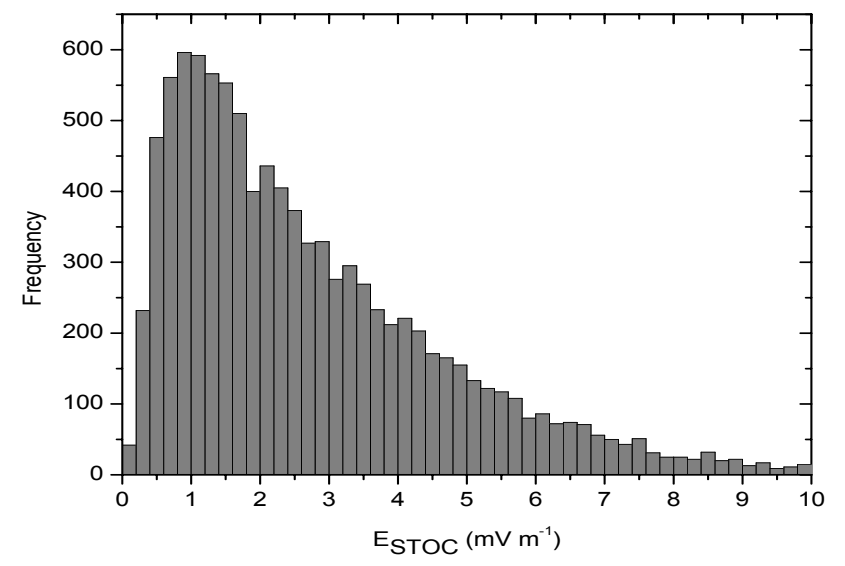

Fig. 2. Distribution of the stochastic electric field in Saturn's inner magnetosphere.

The magnetic force acts on the distribution of charged particles characterized by an initial velocity and an initial energy. The initial velocity includes the corotation velocity $\boldsymbol{v}_{\mathrm{C}}$ and the velocity of a distribution. If the particles come from the planetary atmospheres or ionospheres a Maxwellian velocity distribution, $v_{\mathrm{M}}$, might be assumed. But, for particles coming from the icy-satellites the distribution function can be more exotic (e.g. bi-Maxwellian, Lorentzian, or Kappa). In this work we can take a Maxwellian velocity distribution function in each coordinate for all the cases without loss of generality. Thus, we have:

$\boldsymbol{v}_{1}=\boldsymbol{v}_{\mathrm{C} 1}+\boldsymbol{v}_{\mathrm{M} 1}$

In Eq. (8) the sub-index 1 is referred to the initial value. Using the Monte Carlo method we randomly select one particle which follows a Maxwellian distribution. For this case we employ the Box-Müller Method (1958). Thus, we have:

$v_{\mathrm{M} 1}\left(\chi_{1}, \chi_{2}\right)=\sqrt{\frac{k_{B} T}{m}} \cos \chi_{1} \sqrt{-2 \log \chi_{2}}$

where $\chi_{1}$ and $\chi_{2}$ are random numbers in the interval $[0,1], k_{\mathrm{B}}$ is the Boltzmann's constant, $T$ is the temperature of the plasma source, and $m$ is the mass of the particle. In Table 2 we show the basic characteristics of the main sources of particles in Saturn's inner magnetosphere, which are, the position, the ionic species, the mass, the temperature, and the corotation velocity. For this study we choose Saturn's ionosphere (Moore \& Mendillo 2005), two rings, and three satellites as some examples of sources of neutrals and ions.

In Table 2, we have selected the C-Ring because it is close to the planet and the E-Ring because it is the most extended and this is an important source of neutrals (Jurac et al. 2001). On the other hand, absorption signatures have been observed during the crossings of the L-shells of Janus $\left(\sim 2.5 R_{\mathrm{S}}\right)$, Mimas $\left(\sim 3.1 R_{\mathrm{S}}\right)$ and Enceladus $\left(\sim 4 R_{\mathrm{S}}\right)$, this last one has been chosen because the magnetospheric dynamics may be affected by the rate at which fresh ions are created at such a moon (Paranicas et al. 2005; Kivelson 2006). These signatures do weaken with increasing distance from Saturn, hence the varying strengths of the absorptions. For example, some microsignatures have been observed at the orbit of Dione $\left(\sim 6.3 R_{\mathrm{S}}\right)$ and reported by Simpson et al. (1980) and, recently, Cassini has detected them at the orbit of Tethys ( $\left.\sim 4.8 R_{\mathrm{S}}\right)$ (Roussos et al. 2005). The effect of the corotation velocity on the energization of particles is an interesting task to investigate, for example in Dione's and Rhea's orbits.

The initial kinetic energy for the distribution of particles is $K_{1}$. Equation (8) and $K_{1}$ are initially calculated for each particle.
Table 2. Main sources of neutrals and ions in Saturn's inner magnetosphere. The choices for rings and satellites are explained in the text.

\begin{tabular}{|c|c|c|c|c|}
\hline Source & $r\left(R_{\mathrm{S}}\right)^{a}$ & $\begin{array}{l}\text { Main ion } \\
\text { species }^{b}\end{array}$ & $T(\mathrm{~K})$ & $\begin{array}{l}v_{\mathrm{C}} \\
\left(\mathrm{km} \mathrm{s}^{-1}\right)\end{array}$ \\
\hline $\begin{array}{l}\text { Saturn's } \\
\text { ionosphere }\end{array}$ & $\begin{array}{l}1.2 \\
\text { (north } \\
\text { pole) }\end{array}$ & $\mathrm{H}^{+}, \mathrm{H}_{2}^{+}$ & $\begin{array}{l}400- \\
800^{d}\end{array}$ & 0 \\
\hline C-Ring & $\begin{array}{l}1.705 \\
\text { (above) }\end{array}$ & $\begin{array}{l}\mathrm{H}^{+}, \mathrm{N}^{+}, \mathrm{O}^{+} \text {and } \\
\text { water products }\end{array}$ & $93^{e}$ & 13.64 \\
\hline Enceladus & $\begin{array}{l}4.074 \\
\text { (south } \\
\text { pole) }\end{array}$ & same as C-Ring & $85^{f}$ & 38.98 \\
\hline Dione & 6.576 & same as C-Ring & $87.15^{g}$ & 32.08 \\
\hline E-Ring & $\begin{array}{l}7.665 \\
\text { (above) }\end{array}$ & $\begin{array}{l}\mathrm{H}^{+}, \mathrm{O}^{+} \text {and } \\
\text { water products }\end{array}$ & $89.09^{h}$ & 75.03 \\
\hline Rhea & 9.552 & same as E-Ring & $76.15^{i}$ & 31.26 \\
\hline
\end{tabular}

${ }^{a}$ The distances are calculated through: $r=\sqrt{x^{2}+y^{2}+z^{2}}$. Where $x$ is the orbital distance measured from Saturn's center of mass, $z$ is the distance perpendicular to the orbital plane and $y$ completes the ortogonal coordinate system.

${ }^{b}$ The masses are given in amu $\left(1 \mathrm{amu}=1.66 \times 10^{-27} \mathrm{~kg}\right): \mathrm{H}=1.01$; $\mathrm{H}_{2}=2.02 ; \mathrm{H}_{2} \mathrm{O}=18.02 ; \mathrm{N}=14.01 ; \mathrm{O}=15.99 ; \mathrm{OH}=17.01$.

${ }^{c}$ Water products include $\mathrm{H}_{2} \mathrm{O}^{+}$and $\mathrm{OH}^{+}$.

${ }^{d}$ Atreya et al. (1986).

${ }^{e}$ The brightness temperature has been determined by Murphy (1973). Recently Cassini has observed a temperature asymmetry between the unlit and lit sides of the rings (Spilker et al. 2006).

${ }^{f}$ Spencer et al. (2006); Loeffler et al. (2006); Pontius \& Hill (2006).

${ }^{g}$ Soderblom \& Johnson (1982).

${ }^{h}$ It corresponds to the effective temperature considering that the E-Ring is mainly composed by icy-particles with an albedo of 0.05 .

${ }^{i}$ It is the sunlit temperature on Rhea's surface (Murdin 2001).

\subsubsection{The gravitational acceleration}

We calculate the interaction between the particle and Saturn due to the gravitational attraction. In the last term of Eq. (1) we have expressed the acceleration produced by this force where $G$ is the gravitational constant and $M_{\mathrm{S}}$ is Saturn's mass $\left(5.69 \times 10^{26} \mathrm{~kg}\right)$.

\subsubsection{A collisional term?}

It is important to determine the collisional or non-collisional behavior of Saturn's inner magnetosphere. For this we simulate a spherical magnetosphere whose radius is $10 R_{\mathrm{S}}(6.03 \times$ $10^{8} \mathrm{~m}$ ) and we analyze the mean free path, $\lambda$, between particles within this region. We consider the following three cases: a) collisions between ions and neutrals; b) collisions between neutrals; and c) collisions between ions. For the first case we employ the ionic radius $r_{\mathrm{i}}$ to define the cross section $\sigma$ as: $\lambda=\frac{1}{n \sigma}=\frac{1}{4 \pi r_{\mathrm{i}}^{2} n}$ where $n$ is the observed density in the magnetosphere. In the second case we take the atomic radius $r_{\mathrm{a}}$ to define the cross section. In the third case the dispersion generated by Coulomb interactions must be considered, hence we calculate the impact parameter, $b$, given by $b=\left(\frac{Z e^{2}}{4 \pi \varepsilon_{0} K}\right) \cot \left(\frac{\theta}{2}\right)$ to define the cross section $\sigma$ as: $\lambda=\frac{1}{n \sigma}=\frac{1}{n \pi b^{2}}$ where $n$ is the observed density in the magnetosphere, $Z$ is the atomic number, $e$ is the electric charge, $\varepsilon_{0}$ is the permittivity constant in the vacuum, $K$ is the particle's energy, and $\theta$ is the dispersion angle. In this work we assume a $90^{\circ}$ collision between like particles (ion-ion).

The mean free path between ions ranges from $10^{17}$ to $10^{20} \mathrm{~m}$; the mean free path between neutrals ranges from $10^{7}$ to $10^{8} \mathrm{~m}$, and the mean free path between neutrals and ions 
$<6 \times 10^{8} \mathrm{~m}$. Those values are of the same order or higher than the size of the simulated inner magnetosphere and we conclude that the plasma is non-collisional; hence we do not include a collisional term in Eq. (1).

\subsection{The final energy and the final velocity for the distribution of particles}

In Eq. (1) we are implicitly considering the total force that acts on each particle. In this way we can employ the work-energy theorem to establish:

$\Delta W=F \cdot \mathrm{d} r=F_{x} \Delta x+F_{y} \Delta y+F_{z} \Delta z=\Delta K$.

In Eq. (10) we are calculating the gain (or loss) of kinetic energy after each interaction with the total force (stochastic, Lorentz and gravitational) by each particle in every spatial coordinate $F_{x}, F_{y}$, and $F_{z}$.

The final kinetic energy, $K_{2}$, by particle is given by:

$K_{2}=K_{1}+\Delta K-P$

where $P$ is the loss due to the electromagnetic radiation.

The final velocity $v_{2}$ reached by a charged particle after one interaction is calculated from the expression:

$v_{2}= \pm \sqrt{\frac{2 K_{2}}{m}}$.

In Eq. (12), the sign is assigned through the numerical algorithm by taking into account the initial velocity, the magnitude of the total force and the loss or gain of energy.

The final position in the radial direction reached by a particle after each interaction with the total force is given by the following expression:

$r_{2}=r_{1}+\Delta r$.

In Eq. (13) the initial position is the place occupied by the particle at the end of the previous interaction with the stochastic electric field and $\Delta r$ is a fixed numerical step $\left(1 \times 10^{-3} \mathrm{~m}\right)$.

Equations (11)-(13) are calculated for a given particle after one interaction with the total force. It is important to clarify that such final values become the initial conditions for the next interaction.

Once the selected particle undergoes the chosen number of interactions another particle is randomly injected into the magnetosphere. This procedure finishes when the sample of particles has undergone the same number of interactions. Finally to assure that the particles remain within the inner magnetosphere we establish the cut-off condition: $1 R_{\mathrm{S}}<r<10 R_{\mathrm{S}}$. When $r$ takes a value out of this range the particle is considered as lost.

\section{Results and discussion}

Applying Eq. (6) with the values shown in Table 1, we obtain the stochastic electric field (Fig. 2) that interacts with a particular ion sample (Young et al. 2005). We report the results based on Voyager 1 magnetic field measurements.

The particles are initially characterized by an adequate Maxwellian velocity distribution and its kinetic energy distribution. The sample of particles is located at several sites within Saturn's inner magnetosphere (Table 2).

After the sample of particles interact with the stochastic electric field, in average they tend to gain kinetic energy after some seconds, as we can see in Figs. 3-8.

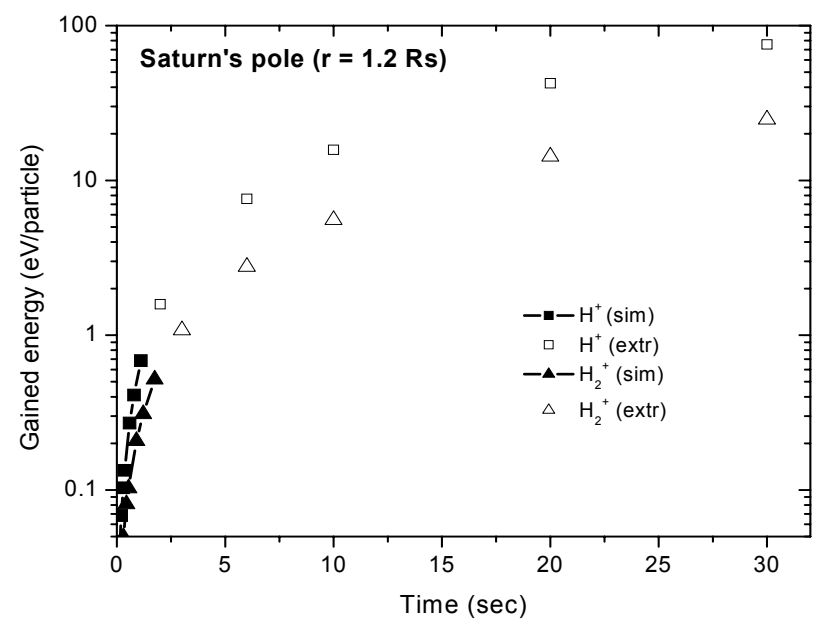

Fig. 3. Profile of the gained energy per particle averaged over the entire distribution against the time for protons and $\mathrm{H}_{2}^{+}$coming from Saturn's ionosphere.

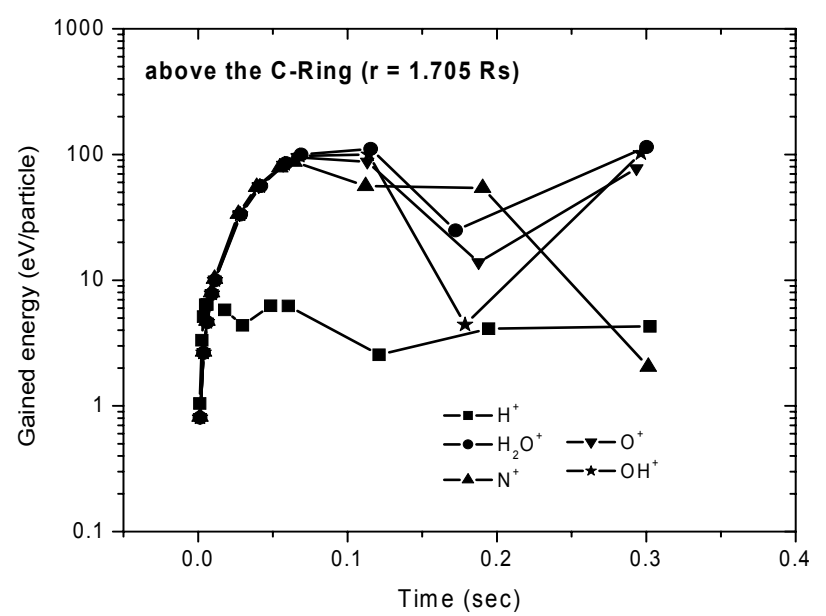

Fig. 4. Profile of the gained energy per particle averaged over the entire distribution against the time for protons and heavy ions coming from the C-Ring.

We observe that protons tend to gain more energy than the heavy ions $\left(\mathrm{H}_{2}^{+}, \mathrm{H}_{2} \mathrm{O}^{+}, \mathrm{N}^{+}, \mathrm{O}^{+}, \mathrm{OH}^{+}\right)$. Additionally, the particles located by the outer part of the inner magnetosphere (for example, Rhea) gain more energy than the particles located near the planet (Saturn's pole). Thus, we can identify different energization regions in Saturn's inner magnetosphere: 1) low energy region (Saturn's pole and Rhea); 2) unstable region (CRing); 3 ) intermediate energy region (Dione); and 4) high energy region (Enceladus and E-Ring).

A possible explanation to this effect is explained in terms of the corotation energy available in each region. After subtracting the final kinetic energy to the initial kinetic energy three terms appear in the energy equation:

$$
\begin{aligned}
\Delta K= & K_{2}-K_{1}=\frac{1}{2} m\left[v_{2}^{2}-v_{1}^{2}\right] \\
= & \frac{1}{2} m\left[\left(v_{\mathrm{D} 2}+v_{\mathrm{C} 2}\right)^{2}-\left(v_{\mathrm{M} 1}+v_{\mathrm{C} 1}\right)^{2}\right] \\
= & \frac{1}{2} m\left(v_{\mathrm{D} 2}^{2}-v_{\mathrm{M} 1}^{2}\right)+\frac{1}{2} m\left(v_{\mathrm{C} 2}^{2}-v_{\mathrm{C} 1}^{2}\right) \\
& +\frac{1}{2} m\left(2 v_{\mathrm{D} 2} v_{\mathrm{C} 2}-2 v_{\mathrm{M} 1} v_{\mathrm{C} 1}\right) .
\end{aligned}
$$




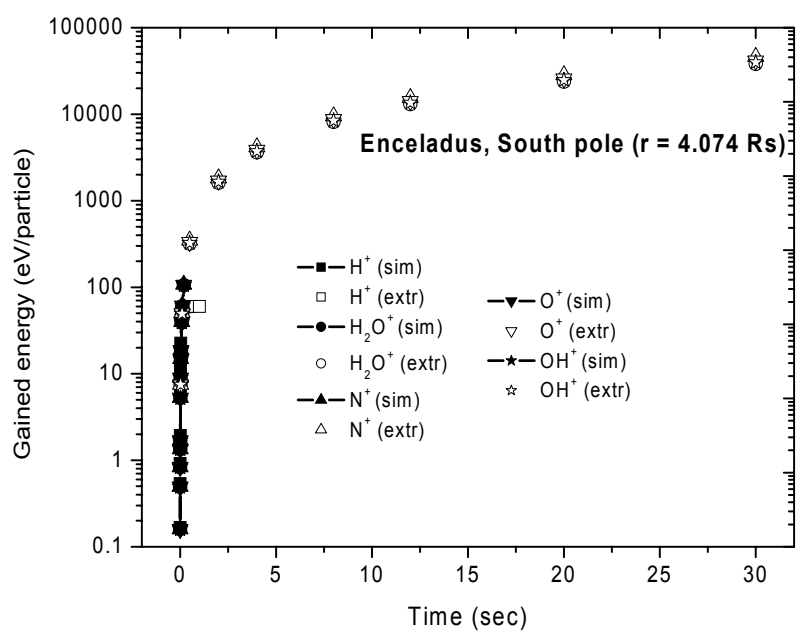

Fig. 5. Profile of the gained energy per particle averaged over the entire distribution against the time for protons and heavy ions coming from Enceladus.

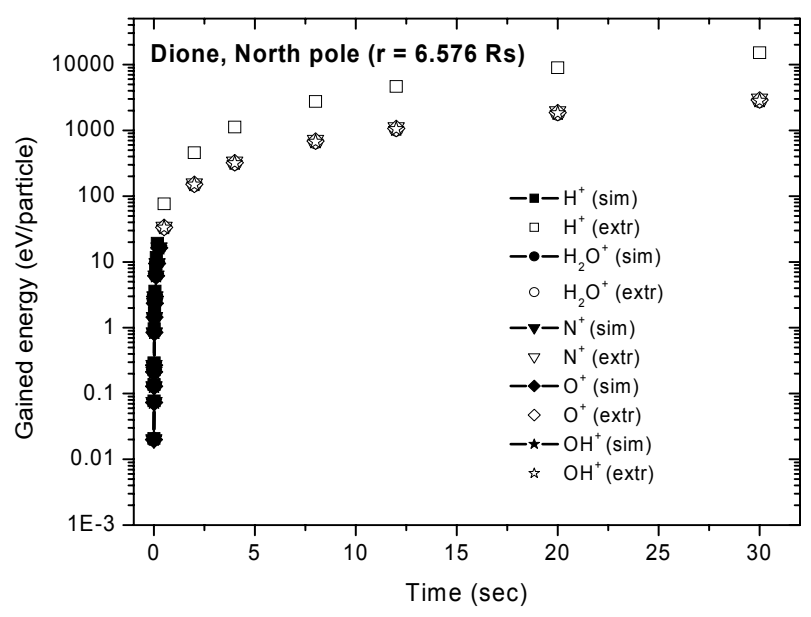

Fig. 6. Profile of the gained energy per particle averaged over the entire distribution against the time for protons and heavy ions coming from Dione.

The first term in Eq. (14) is the distribution energy where $v_{\mathrm{D} 2}$ is the final velocity distribution and $v_{\mathrm{M} 1}$ is the initial Maxwellian velocity distribution; the second term is the corotational energy where $v_{\mathrm{C} 2}$ is the final corotation velocity and $v_{\mathrm{C} 1}$ is the initial corotation velocity and the third term is a mixture of both types of energy, distribution and corotational. In the inner magnetosphere this last term is larger than the first term as the particles move away from the planet and the stochastic mechanism becomes more effective in specific places (Fig. 9).

In the referenced figures we also show some values derived from an extrapolation based on the form of the curves by taking into account the mathematical function that better fits them and proceed with the appropriate goodness-of-fit hypothesis test. For example, after $30 \mathrm{~s}$, protons coming from Saturn's ionosphere can reach energies of some hundreds of eV (Fig. 3), by contrast, protons coming from Dione and Rhea (Figs. 6 and 8) can reach energies of some tens of keV. For comparison in Fig. 10 we show the gained energy for protons coming from the sources shown in Table 2. The values are normalized to the minimum energy for a proton in the inner magnetosphere, namely, $0.1049 \mathrm{eV}$.

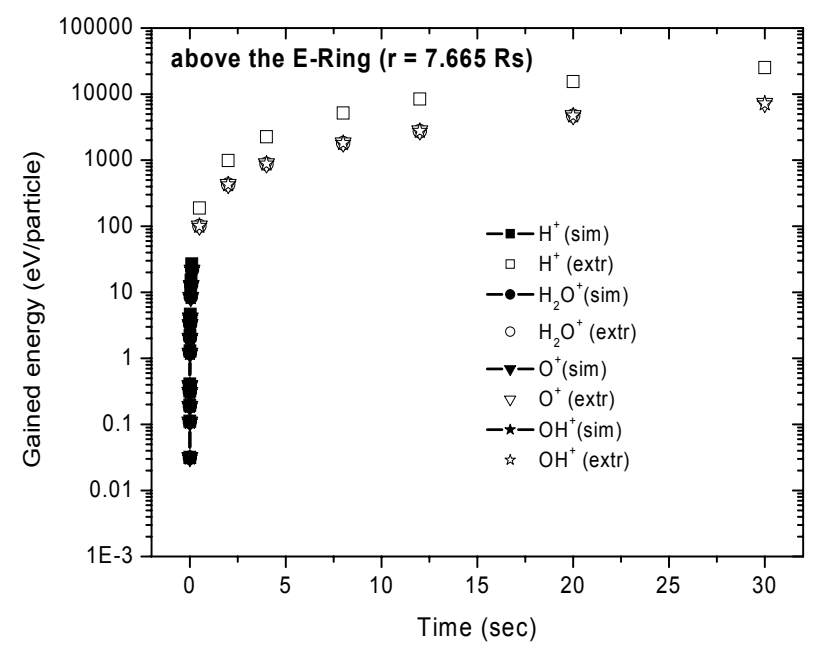

Fig. 7. Profile of the gained energy per particle averaged over the entire distribution against the time for protons and heavy ions coming from the E-Ring.

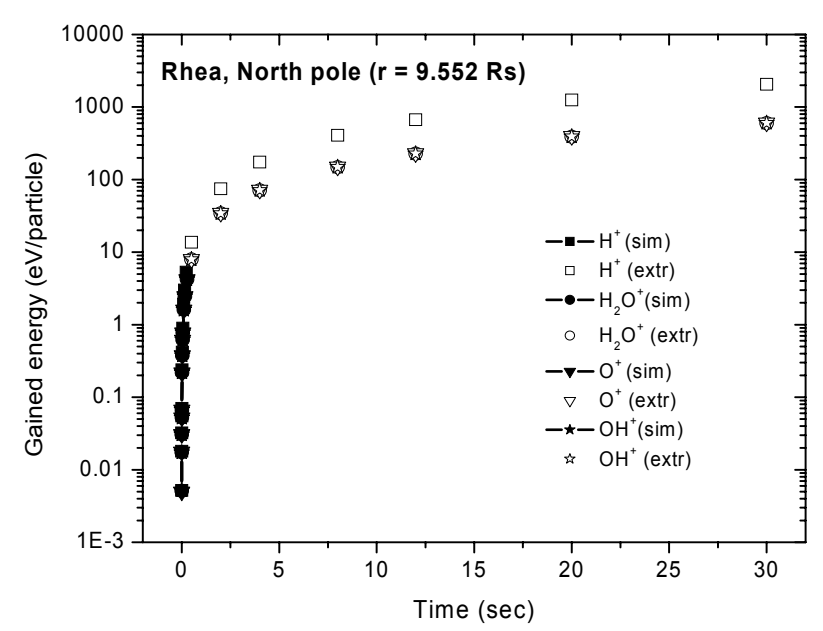

Fig. 8. Profile of the gained energy per particle averaged over the entire distribution against the time for protons and heavy ions coming from Rhea.

It is evident from Fig. 10 that protons are being energized more at Enceladus and this result compares well with the stochastic energy curve shown in Fig. 9.

In all the cases we examined a power-law function is the best fit. However, in Fig. 4 we observe that protons and ions located above the C-Ring show a different tendency, it is a region where particles can gain or lose energy. Near the orbit of Enceladus, protons seem to be described by a Logistic function.

For results obtained directly from the numerical simulation we observe that the Maxwellian velocity distribution has preserved its shape after the acceleration process, except for particles coming from the C-Ring and protons coming from Enceladus. To prove this fact we do a goodness-of-fit hypothesis test for each case and considering the Jarque-Bera statistical criterion with the $95 \%$ significance level.

At lower energies the ion spectra observed by Voyager 1 is described well by a Maxwellian distribution and at higher energies by a power-law (Krimigis et al. 1981, 1982). In these spectra, the wide variety of energies for electrons, protons and heavy ions can indicate the action of other acceleration mechanisms. In the present model, a sample of ions interacts with a 


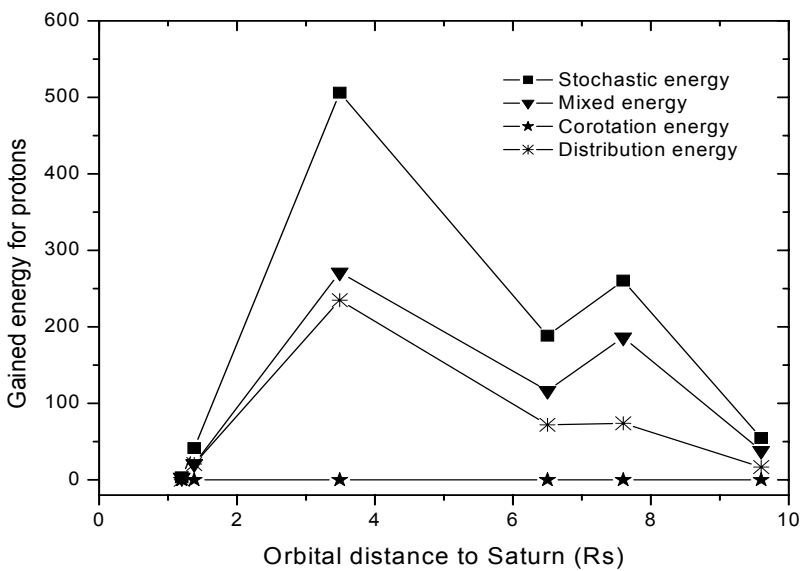

Fig. 9. Gained energy against distance to Saturn for protons coming from different sources in the inner magnetosphere. The gained energy is normalized to the minimum value of the initial energy, namely, $0.1049 \mathrm{eV}$.

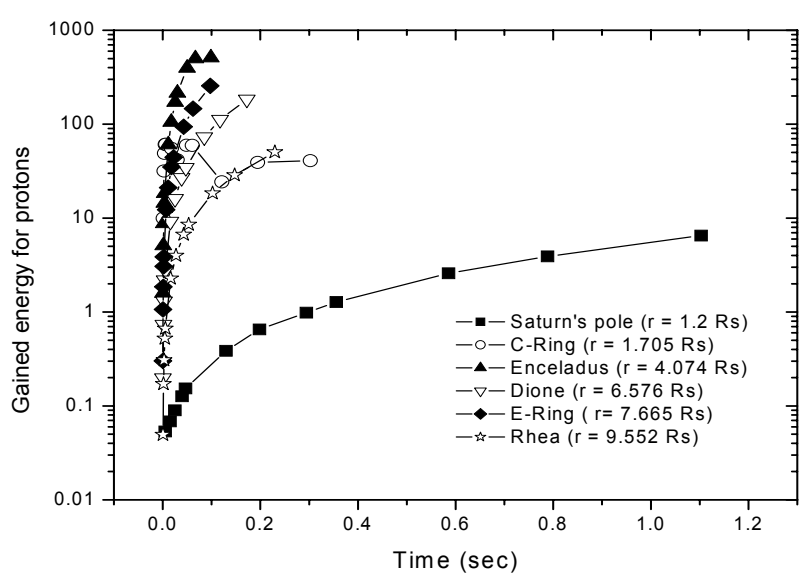

Fig. 10. Comparison of the gained energy against the time for protons coming from the sources shown in Table 2 . The values are normalized to the minimum energy for a proton in the inner magnetosphere. Only values obtained directly from the simulation are shown.

stochastic electric field gaining energy on average over time, thus, we can not directly compare our spectra with the observations. The proposed model provides a helpful insight to the energization problem in Saturn's magnetosphere.

Figures 11-13 show the spectrum for the final energy reached by a sample of protons coming from Saturn's north pole, the vicinity of Mimas (where the particles have been previously accelerated) and Rhea.

In the first case for particles with energies $\left(0.6 \mathrm{eV}<E_{\mathrm{p}}<\right.$ $2 \mathrm{eV}$ ) the spectrum follows an asymmetric sigmoidal function, in the second case the high-energy part of the spectrum $(50 \mathrm{keV}<$ $E_{\mathrm{p}}<150 \mathrm{keV}$ ) is described through a power law distribution (power index $=-2.38$ ), and for the third case we find two different distributions: logistic $(E<10.4 \mathrm{eV})$ and an exponential decay $(E>10.6 \mathrm{eV})$.

The component of the final velocity in the $y$-direction shows a bimodal feature for particles located on the north pole (Fig. 14). This structure can be explained in terms of the theoretical studies conducted by Durand-Manterola (2003). In that study the author establishes that the probability gaining energy is always larger than the probability losing energy. Thus, during the acceleration process particles with low linear momentum

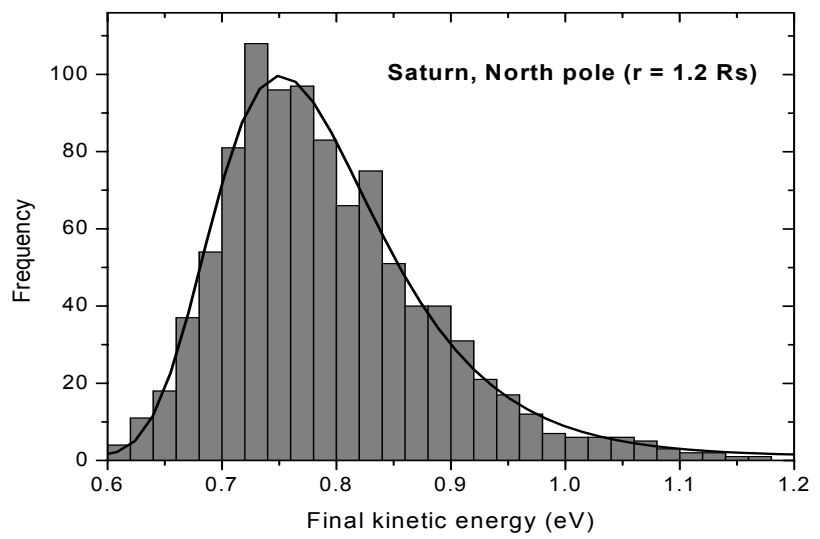

Fig. 11. Histogram of frequencies for the final kinetic energy reached by a sample of protons after $5 \times 10^{7}$ interactions and its fitting function in Saturn's north pole. The particles gain $\sim 1 \mathrm{eV}$ after $1 \mathrm{~s}$ and the energy spectrum is well-described by an asymmetric sigmoidal function.

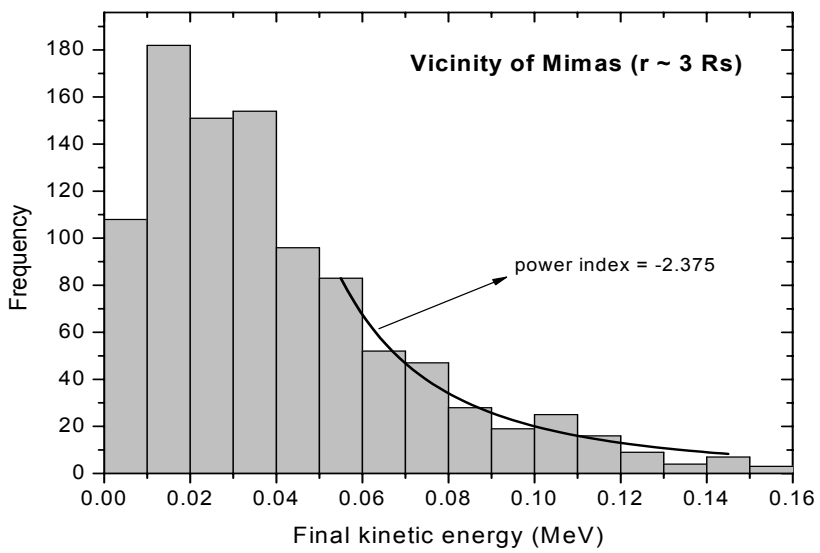

Fig. 12. Histogram of frequencies for the final kinetic energy reached by a sample of protons after $5 \times 10^{7}$ interactions and its fitting function in the vicinity of Mimas. Initially the particles have an energy of $0.02 \mathrm{MeV}$ and after 5 milliseconds the energy spectrum can be described by a power-law with an index of -2.38 for $E>0.06 \mathrm{MeV}$.

(north pole) are being pumped from the center of the distribution to higher speeds as a result of their own acceleration, by contrast, particles with larger linear momentum (Rhea) do not show the bimodal feature.

\section{Conclusions}

We have developed a model to explain the energization of particles in Saturn's inner magnetosphere. Basically this model assumes that the action of a stochastic electric field on a sample of particles is the main cause of the energization. The stochastic electric field is obtained through the Maxwell's equations and Voyager 1 magnetic field fluctuations.

Saturn's inner magnetosphere is simulated by taking into account its symmetry and its non-collisional behavior. We have assumed that is populated by protons and heavy ions $\left(\mathrm{H}_{2} \mathrm{O}^{+}, \mathrm{N}^{+}\right.$, $\mathrm{O}^{+}$, and $\mathrm{OH}^{+}$) from the primary sources like the rings and the satellites. Without loss of generality the particles are characterized by an initial Maxwellian velocity distribution and the corresponding energy distribution. 


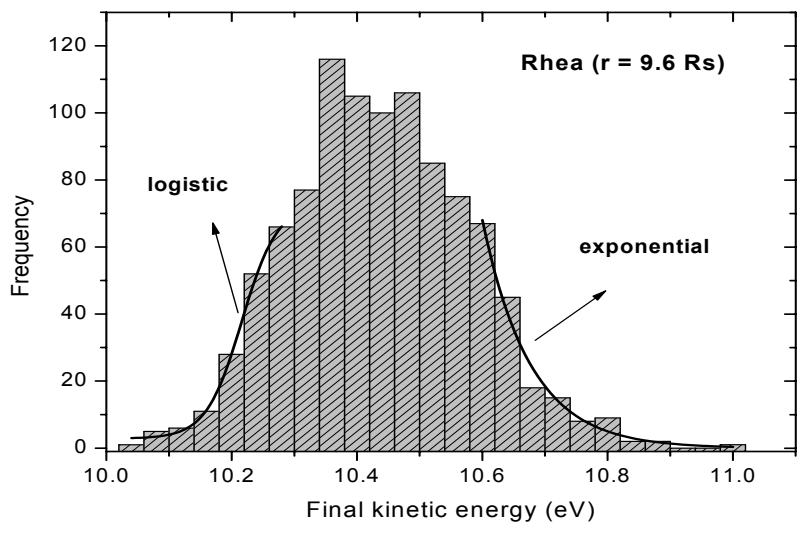

Fig. 13. Histogram of frequencies for the final kinetic energy reached by a sample of protons after $5 \times 10^{7}$ interactions and their fitting functions in Rhea. The energy spectrum is well-described through two different distributions, it is, for $E<10.4 \mathrm{eV}$ a logistic function takes place, and for $E>10.6 \mathrm{eV}$ an exponential decay function is more adequate.

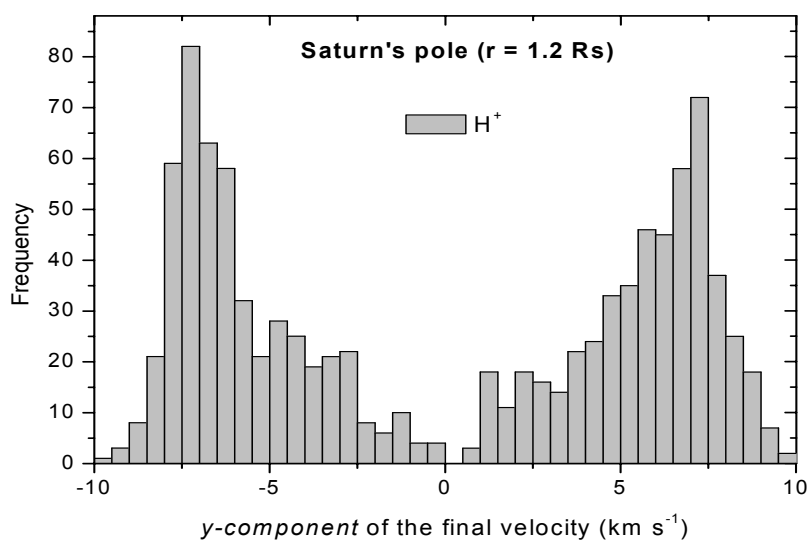

Fig. 14. Histogram of frequencies for the final velocity in the corotation $y$-direction reached by a sample of protons after $5 \times 10^{7}$ interactions in Saturn's north pole.

We can summarize the effects produced by the action of the stochastic electric field on a sample of particles in Saturn's inner magnetosphere as follows:

1. The particles experience - on average - a gain in energy with time. That effect is revealed through an increase in the plasma temperature (stochastic heating) and an increase of the mean velocity (stochastic acceleration).

2. The initial Maxwellian velocity distribution maintains its shape, except for protons and heavy ions located near the $\mathrm{C}$-Ring and protons coming from Enceladus where the velocity distributions become unstable.

3. The bimodal feature observed in the y-component of the final velocity depends on the initial linear momentum (it ranges from $6.85 \times 10^{-24} \mathrm{~kg} \mathrm{~m} \mathrm{~s}^{-1}$ to $2.25 \times 10^{-21} \mathrm{~kg} \mathrm{~m} \mathrm{~s}^{-1}$ ) acquired by the sample of particles. It is, for particles located close to the planet the linear momentum is lesser than for particles located far and, comparing it with the impulse given by the stochastic electric field $\left(5.55 \times 10^{-21} \mathrm{~kg} \mathrm{~m} \mathrm{~s}^{-1}\right)$, the probability of being accelerated is greater.

4. The effectiveness of the mechanism strongly depends on the three terms that appear in the resulting energy equation. The first term is related to the distribution energy, the second term is due to the corotational energy and the third term involves the distribution and the corotational energies. This last term is larger when the particles move away from the planet. Thus, the proposed stochastic mechanism is more effective in some places within the inner magnetosphere.

5. As a consequence of the fact described in point 4 we can distinguish several energization regions in Saturn's inner magnetosphere: 1) low energy region (Saturn's pole and Rhea); 2) unstable region (C-Ring); 3) intermediate energy region (Dione); and 4) high energy region (Enceladus and E-Ring).

6. The mechanism can reinforce the action of other acceleration processes. When we consider a previously energized sample of protons near Mimas, the stochastic mechanism increases their energy in a few milliseconds.

7. This mechanism results in a fast way to produce energetic particles in a few seconds.

8. Through this mechanism it is possible to produce suprathermal tails. The origin of such structures has not been understood at all, thus in future work we will extend the calculations for a longer time in order to study how these structures can form.

9. The work presented here is based on the single flyby of Saturn by Voyager 1. In a future task we will carry out our simulations using Cassini magnetometer data and then compare with the obtained in this work. Moreover, our results can be tested or validated taking into account the plasma measurements by Cassini. In that sense, we expect that the observed density of ions/neutrals show a peak in its distribution at the places where the stochastic mechanism is less effective $\left(\sim 1.3 R_{\mathrm{S}}, \sim 6.5 R_{\mathrm{S}}\right.$ and $\left.\sim 9.6 R_{\mathrm{S}}\right)$. In such places the particles do not have enough energy to leave the region and then, they can stay there longer until the escape energy is reached. In addition, the measured plasma temperature could show a low value because of the energization. By contrast, in the places where the stochastic mechanism is more effective $\left(\sim 3.5 R_{\mathrm{S}}\right.$ and $\left.\sim 7.6 R_{\mathrm{S}}\right)$ the density of ions/neutrals is low and the plasma temperature is high.

\section{References}

Abe, T., \& Nishida, A. 1986, J. Geophys. Res., 91, 10003

Atreya, S. K., White J. H. Jr., Donahue, T. M., et al. 1986, in Saturn, ed. T. Gehrels, \& M. S. Matthews, 239

Barbosa, D. 1986, J. Geophys. Res., 91, 5605

Blandford, R. D. 1994, ApJSS, 90, 515

Box, G. E. P., \& Müller, M. E. 1958, Annals Math. Stat., 29, 610

Bridge, H. S., Belcher, J. W., Lazarus, A. J., et al. 1981, Science, 212, 217

Bridge, H. S., Bagenal, F., Belcher, J. W., et al. 1982, Science, 215, 563

Durand-Manterola, H. 2003. Acceleration of particles in a random force field, In IX Meeting of the Dynamic Fluids Division Proceedings, Sociedad Mexicana de Física, 142

Frank, L. A., Burek, B. G., Ackerson, K. L., et al. 1980, J. Geophys. Res., 85, 5695

Fillius, W., \& McIlwain, C. E. 1980, J. Geophys. Res., 85, 5803

Gladstone, G. R., Waite, J. H., Grodent, D., et al. 2002, Nature, 415(6875), 1000

Grodent, D., Gérard, J.-C., Clarke, J. T., et al. 2004, J. Geophys. Res., 109(A5), CiteID A05201

Grodent, D. 2004, EOS Trans AGU 85(17), Joint Assembly Suppl., Abstract SM12A-04

Jurac, S., Johnson, R. E., \& Richardson, J. D. 2001, Icarus, 149, 384

Jurac, S., McGrath, M. A., Johnson, R. E., et al. 2002, Geophys. Res. Lett., 29(24), 25-1

Kivelson, M. G. 2005, Adv. Space Res., 36, 2077

Kivelson, M. G. 2006, Science, 311, 1391

Kivelson, M. G., \& Russell, C. T. 1997, in Introduction to Space Physics, 165

Krimigis, S. M., \& Armstrong, T. P. 1982, Geophys. Res. Lett., 9, 1143

Krimigis, S. M., Armstrong, T. P., Axford, W. I., et al. 1981, Science, 212(10), 225 
Krimigis, S. M., Armstrong, T. P., Axford, W. I., et al. 1982, Science, 215, 571 Krimigis, S. M., Carbary, J. F., Keath, E. P., et al. 1983, J. Geophys. Res., 88, 8871

Krimigis, S. M., Mitchell, D. G., Hamilton, D. C., et al. 2005, Science, 307, 1270 Krupp, N. 2005, Space Sci. Rev., 116, 345

Loeffler, M. J., Raut, U., \& Baragiola, R. A. 2006, ApJ, 649, L133

Mauk, B. H., Saur, J., Mitchell, D. G., et al. 2005, Geophys. Res. Lett., 32, L14S05

Mitchell, D. G., Brandt, P. C., Roelof, E. C., et al. 2005, Geophys. Res. Lett., 32, L20S01

Moore, L., \& Mendillo, M. 2005, J. Geophys. Res., 110, A05310

Murdin, P. 2001, in Encyclopedia of Astronomy and Astrophysics, article 4439

Murphy, R. E. 1973, ApJ, 181, L87

Ness, N. F., Acuña, M. H., Lepping, R. P., et al. 1981, Science, 212, 211

Ness, N. F., Acuña, M. H., Behannon, K. W., et al. 1982, Science, 215, 558

Paranicas, C., Mitchell, D. G., Livi, S., et al. 2005, Geophys. Res. Lett., 32, L20101

Pontius, D. H., \& Hill, T. W. 2006, J. Geophys. Res., 111(A9), CiteID A09214

Richardson, J. D. 1986, J. Geophys. Res., 91, 1381

Richardson, J. D., Eviatar, A., \& Siscoe, G. L. 1986, J. Geophys. Res., 91, 8749
Roussos, E., Krupp, N., Woch, J., et al. 2005, Geophys. Res. Lett., 32, L24107

Schardt, A. W., Lepping, R. P., Kurth, W. S., \& Maclennan, C. G. 1985, J. Geophys. Res., 90, 8539

Simpson, J. A., Bastian, T. S., Chenette, D. L., et al. 1980, J. Geophys. Res., 85, 5731

Sittler Jr., E. C., Ogilvie, K. W., \& Scudder, J. D. 1983, J. Geophys. Res., 88, 8847

Sittler Jr., E. C., Johnson, R. E., Smith, H. T., et al. 2006, J. Geophys. Res., 111, A09223

Stephens, M. A. 1974, J. Am. Stat. Assoc., 69, 730

Soderblom, L. A., \& Johnson, T. V. 1982, Scient. Am., 246, 101

Spencer, J. R., Pearl, J. C., Segura, M., et al. 2006, Science, 311 (5766), 1401

Spilker, L. J., Pilorz, S. H., Ferrari, C., et al. 2006. Cassini CIRS observations of thermal differences in Saturn's main rings with increasing phase angle. In 37th Lunar and Planetary Science

Treumann, R. A., \& Pottelette, R. 2002, Adv. Space Res., 30(7), 1623

Wahlund, J. E., Boström, R., Gustafsson, G., et al. 2005, Geophys. Res. Lett., 32, L20S09

Young, D. T., Berthelier, J.-J., Blanc, M., et al. 2005, Science, 307, 1262 